\title{
WORKPAD: 2-Layered Peer-to-Peer for Emergency Management through Adaptive Processes
}

\author{
(Invited Paper) \\ Tiziana Catarci, Fabio De Rosa, Massimiliano de Leoni, Massimo Mecella*, \\ Michele Angelaccio ${ }^{\dagger}$, Schahram Dustdar ${ }^{\ddagger}$, Begoña Gonzalvez ${ }^{\S}$, Giuseppe Iiritano , \\ Alenka Krek", Guido Vetere**, Zdenek M. Zalis ${ }^{\dagger \dagger}$ \\ *Università di Roma "La Sapienza", Italy \\ Email: \{derosa,deleoni,catarci,mecella\}dis.uniromal.it \\ †Università di Roma "Tor Vergata", Italy \\ Email: angelaccio $a$ disp.uniroma2.it \\ $¥$ Technische Universitaet Wien, Austria \\ Email: dustdar@infosys.tuwien.ac.at \\ ${ }^{\S}$ Moviquity, Spain \\ Email: bgr@moviquity.com \\ TRegione Calabria - Dipartimento della Protezione Civile, Italy \\ Email: g.iiritano@protezionecivilecalabria.it \\ "Salzburg Research, Austria \\ Email: alenka.krek@salzburgresearch.at \\ ${ }^{* *}$ IBM Italia, Italy \\ Email: gvetere@it.ibm.com \\ ${ }^{\dagger \dagger}$ Software602, Czech Republic \\ Email: zalis@software602.cz
}

\begin{abstract}
In this paper, we present a recently funded European research project, namely WORKPAD, that aims at designing and developing an innovative software infrastructure (software, models, services, etc.) for supporting collaborative work of human operators in emergency/disaster scenarios. In such scenarios, different teams, belonging to different organizations, need to collaborate with one other to reach a common goal; each team member is equipped with handheld devices (PDAs) and communication technologies, and should carry on specific tasks. In such a case we can consider the whole team as carrying on a process, and the different teams (of the different organizations) collaborate through the "interleaving" of all the different processes (macroprocess). Each team is supported by some back-end centre, and the different centres need to cooperate at an inter-organizational level to reach an effective coordination among teams. The project will investigate a 2-level framework for such scenarios: a backend peer-to-peer community, providing advanced services requiring high computational power, data $\&$ knowledge $\&$ content integration, and a set of front-end peer-to-peer communities, that provide services to human workers, mainly by adaptively enacting processes on mobile ad-hoc networks.
\end{abstract}

\section{INTRODUCTION}

The widespread availability of network-enabled handheld devices (e.g., PDAs with WiFi/UMTS capabilities, TETRA smart terminals, etc.) has made the development of pervasive computing environments an emerging reality, very suitable for managing emergency/disaster situations.
Disaster is a broad term; for the purpose of the work presented in this paper, disasters can be defined as a serious disruption of the functioning of a community or a society causing widespread human, material, economic or environmental losses which exceed the ability of the affected community or society to cope using its own resources [1].

The total systematic coordination activities for the prevention and respectively the coverage of natural and man-made disasters are termed as disaster management activities. These activities can be grouped into five phases, as suggested by [3], [4]. They are structured by time and function for all types of disasters (see Figure 1). These phases are related to each other and they involve different types of skills.

The preventive measures are divided into planning, mitigation and preparedness activities. During the planning phase it is necessary to analyze and document the possibility of an emergency event or a disaster and the potential consequences or impacts on life, property and environment. The results of this phase are essential for the next preventive phases. Mitigation activities eliminate or reduce the probability of a disaster. It includes long-term activities designed to reduce the effects of unavoidable disasters. In the preparedness phase governments, organizations and individuals, develop plans to save lives and minimize disaster damage. Preparedness measures seek to enhance disaster response operations. 


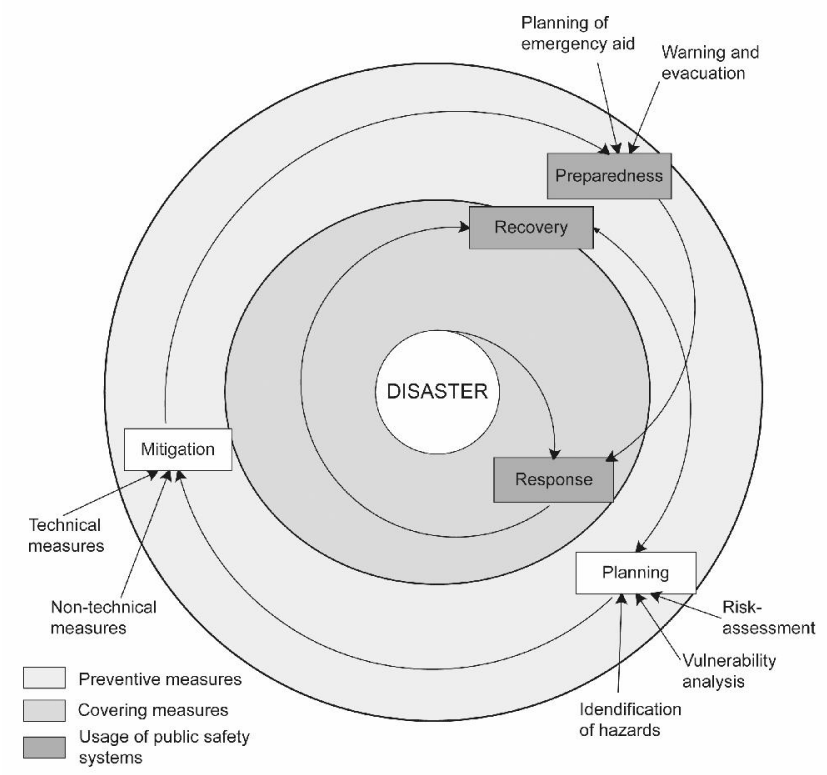

Fig. 1. The life cycle of disaster management [2]

When a disaster or emergency happens, the response activities are designed to provide emergency assistance for victims. They also aim at stabilizing the situation and reducing the probability of secondary damage and speed recovery actions. The recovery activities aim at returning the living conditions to normal or better and they usually include two sets of activities. Short-term recovery activities return vital life-support systems to a minimum operating standard. Long-term recovery activities may continue for a number of years after a disaster. This phase represents also the first step to a new planning phase, because this is the point when the analysis of the cause of the disaster or emergency takes place. WORKPAD, the project presented in this paper, will focus on response and short-term recovery phases, by providing an IT software and communication infrastructure supporting operators. Nevertheless the results achieved can be also exploited in the other phases; of course the response and short-term recovery phases are the more demanding ones (i.e., real-time support should be provided, reliability, etc.) and therefore it should be feasible to apply the achieved results also in the other phases, that present less demanding needs.

Currently, so-called Public Safety Systems (PSSs), typically arranged in specialized organizational centres, are used in three phases within the disaster management. During the preparedness phase governments and organizations provide personal training in how to use the PSS. The main application of these systems is in the response phase, where computer programs give instructions to the rescue teams. In the case of the emergency situation, the emergency call will be accepted and the rescue teams alarmed and controlled. In the recovery phase PSS produce reports, maps, information showing the extent of the damage caused by the disaster. An analysis of the typical architectures of PSSs can be found in [2], in which it is also discussed how current systems are mainly centralized. The comparison shows that they can be used for emergency cases, e.g., traffic accidents or small fires, where the emergency teams need GIS functions to query addresses, to find the way and to map the emergency location. The disadvantage of the compared PSS systems is that they do not use real-time traffic data such as information about the current traffic flow and traffic jams for the network analysis. They are also only partially usable for large emergencies or disasters because the covering activities of these unusual events need systems which can be used directly at the emergency locations. For these requirements it is necessary to develop mobile applications.

The objective of the WORKPAD project is to investigate how to create communities of PSSs and how to integrate them, and how to enable mobile teams to exploit such back-end PSSs through the interplay of MANET technologies, workflow management and geocollaboration.

In complex emergency scenarios, different teams, belonging to different organizations, need to collaborate. Each team member is equipped with handheld devices (PDAs) and communication technologies, and should carry on specific tasks. In such a way we can see the whole team as carrying on a process, and the different teams (of the different organizations) collaborate through the "interleaving" of all the different processes. In turn each team is supported by some back-end center, and, in order to coordinate between teams, the different centers need to cooperate at an inter-organizational level. We can distinguish between a back-end peer-to-peer community, providing advanced services requiring high computational power, and a set of front-end peer-to-peer communities, that provide services to human workers:

- The back-end community is constituted mainly by static/traditional computers, possibly arranged in a GRID, that interact in a P2P fashion. Such services, coarsegrained, require integration of data \& knowledge \& content. The interesting aspect is that the community is inter-organizational (each peer belongs to a certain organization) and each system is enabled to act as service provider, requestor, or integrator. In particular, the integration should be dynamic, flexible, and non-intrusive.

- A single front-end community is constituted by the operators of a team, equipped with mobile devices, connected in an ad hoc and peer-to-peer fashion, that carry on a process, in which the adaptiveness to connection/task anomalies is fundamental

\section{A. A Scenario}

As an example, consider the following scenario of disaster recovery. After an earthquake (or a hurricane), a team (e.g., belonging to the Homeland Security Department), equipped with mobile devices (laptops and PDAs), is sent to the disaster area to evaluate the state of specific sites. Their goal is to document the damage directly on a situation map, and to schedule following activities (e.g., reconstruction jobs). Before this process starts, the team leader has stored all area details, 
including a site map, a list of the most important objects at the site, and some previous reports and materials. All such details have been provided by the back-end centre of the Homeland Security Department, which has constructed them by integrating information $\&$ knowledge $\&$ content stored by many other peer organizations (e.g., the Ministry of Internal Affairs, some basic spatial data provided by different public and private organizations, etc.). It is worth observing that the integration and involvement of a particular peer has been dynamically and adaptively decided on the basis of the specific process, which in turn depends on the given emergency situation. Therefore no pre-existing, defined integration infrastructure exists among the back-end peers, but it is rather dynamically built ondemand.

On the front-end, the team constitutes a MANET (Mobile Ad hoc NETwork), in which the team leader's device coordinates the other team members' devices by providing appropriate information (for example, maps, important objects, and so on) and assigning activities. MANETs are networks of mobile devices that communicate with one another via wireless links without relying on an underlying infrastructure. This distinguishes them from other types of wireless networks for example, cell networks or infrastructure-based wireless networks. To achieve communication in a MANET, each device acts as an endpoint and as a router forwarding messages to devices within radio range. MANETs are a sound alternative to infrastructure-based networks whenever an infrastructure is no longer available, or can't be used, as in emergency scenarios [5]. As an example, consider what happened recently during the Katrina's emergency in New Orleans, USA: among the different communication infrastructures, only the satellitebased one survived, but it was not possible to use it for all communications of the teams working in the area, with the known dramatic consequences. Conversely, teams equipped with PDAs forming a MANET would be able to communicate each other, and exploit a possible satellite channel available to one of them (e.g., the team leader) for possible coordination with other teams.

The team members' devices enable them to execute some operations. Such operations, possibly supported by particular hardware (for example, digital cameras, different communication connections ${ }^{1}$, computational power for image processing, main storage, etc.), are offered as software services to be coordinated. Such a coordination is carried out by a specific coordination layer hosted by the team leader device.

As an example of a possible process, after visual analysis of a building, supported by some map-based application, team member 1 (using his/her device - a PDA) fills out a report and enter attributes and graphic data related to the damage. The team leader will analyze these reports and spatial data, with the help of specific software, to schedule the next activities. Team member 3 takes pictures of the precarious buildings, whereas the team member 2 is in charge of the image processing

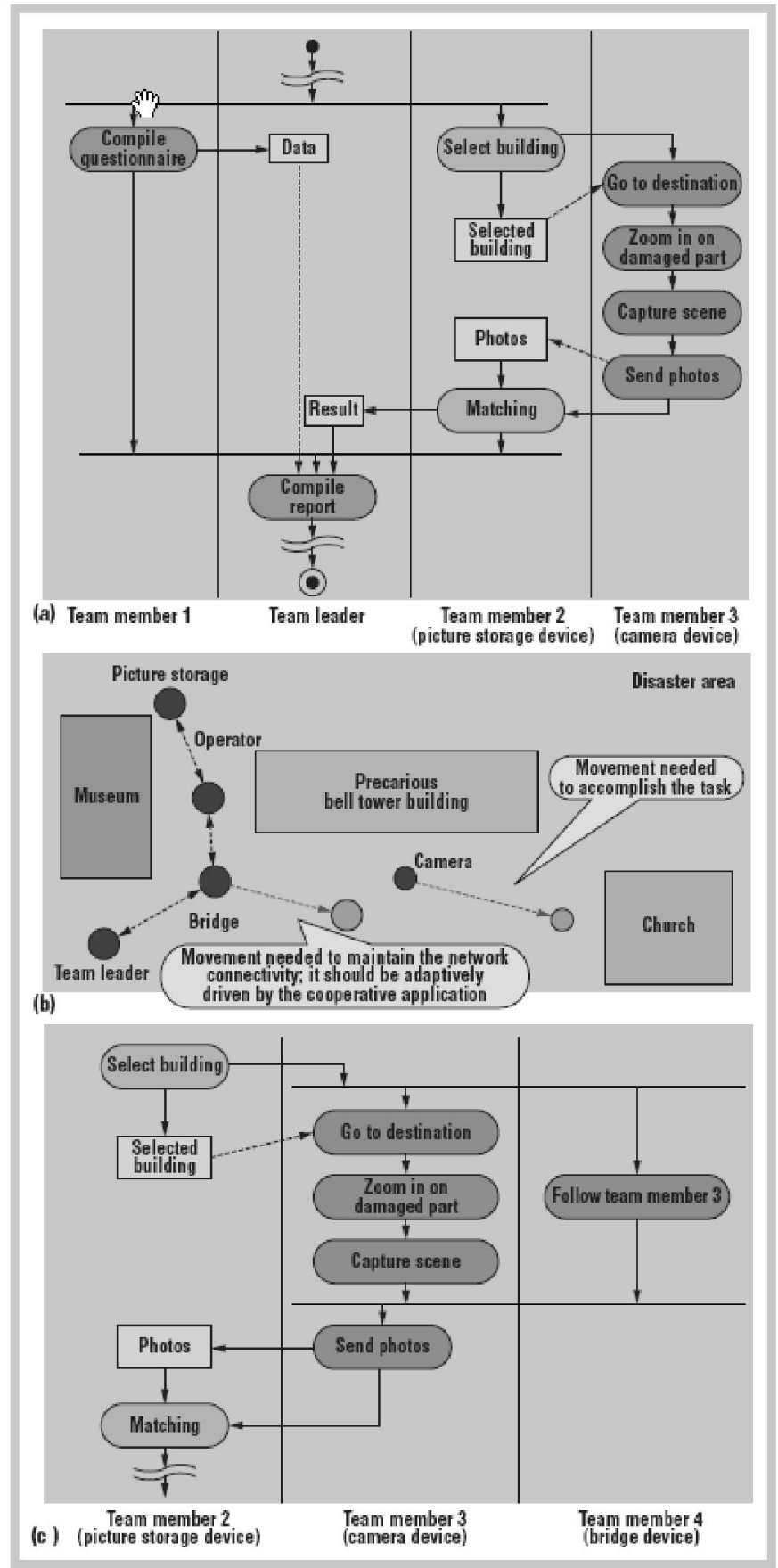

Fig. 2. Disconnection prediction for adaptive coordination in MANETs: (a) the process, (b) a critical situation and adaptive management, and (c) a detail of the process, which has been modified to prevent the disconnection of mobile devices (picture from [5])

\footnotetext{
${ }^{1}$ Depending on the specific situation, communication can be GPRS/UMTSbased, satellite-based, TETRA-based, etc.
} 
of older and recent photos of the site (for example, to initially identify architectural anomalies). The outlined process is shown in Figure 2(a). In this situation, matching new pictures with previous ones might be useful. So, the device/PDA with the high-resolution camera and the device/PDA with the older stored pictures must be connected. But in a scenario such as the one in Figure 2(b), the camera-equipped device/PDA's movement might result in its disconnection from the other devices. A pervasive architecture should be able to predict such situations, to alert the coordination layer. The coordination layer, in turn, would direct a "bridge" device (team member 4 's PDA) to follow the device/PDA that's going out of range, maintaining the connection and ensuring a path between the devices (see Figure 2(c)). In this way, the coordination layer, on the basis of the disconnection prediction, schedules the execution of new, unforeseen activities (note the new activity for team member 4). Such an adaptive change of the process is centrally managed by the coordination layer, which has global knowledge about the status of all the devices and takes into account idle devices, operations that can be safely delayed, and so on.

\section{B. Research Issues}

In order to support such a complex scenario, from the provision of data $\&$ knowledge \& content to front-end teams to their process executions, different research problems should be addressed:

- Devising a 2-layer peer-to-peer architecture, including both the back-end peers and the front-end teams.

- Investigating novel basic techniques for P2P data \& knowledge \& content integration, to be exploited on the back-end.

- Investigating novel adaptive and context-aware techniques for cooperative work and workflow management among mobile devices on the front-end, with attention to usability issues.

- Investigating how to exploit and leverage geo-referenced information, that plays an important role both (i) in the dynamic building of the back-end integration system and (ii) in the adaptive process management on the front-end teams.

- Devising appropriate solutions around emergency communications, wireless communications and robust link (i.e., connecting front-end and back-end) networks (e.g., satellite-based, TETRA-based, etc.), as they are key elements in helping emergency services respond in extreme situations.

\section{Content and Structure of the Paper}

The purpose of this paper is to outline the WORKPAD approach in solving the previous research issues, based on a 2-layered P2P architecture. More specifically, the paper is organized as it follows. Section II introduces the WORKPAD architecture, whereas Section III compares with relevant research work and finally Section IV concludes the paper.

\section{THE WORKPAD ARCHITECTURE}

Figure 3 shows the WORKPAD architecture:

Back-end. By definition, emergencies are largely unpredictable in their occurrence, evolution, and impact. Usually, they involve communities - whose infrastructures, organizations, and supplies are not designed to work together - in new overwhelming circumstances, when cooperation processes and information exchanges must be quickly put in place. To cope with unpredictability, adaptive strategies have been devised by the Italian Homeland Security Agency (see for instance the "Augustus Method" [6]), based on the observation that - as the Emperor Augustus used to say - " the value of a plan decreases as the complexity of the situation grows ". Such strategies are meant to provide a suitable approach to the problem of coordinating complex operations according to flexible plans in emergency contexts. An IT infrastructure able to support a strategy like the "Augustus Method" should be simple, robust, rapid, flexible, dependable, and tolerant: it is easy to see that, from an IT standpoint, these features require the availability of specific infrastructures. WORKPAD's back-end will focus on a peer-to-peer architecture, in which each system can act both as data provider, consumer, and integrator. WORKPAD's back-end back-end infrastructure will allow front-end operators to access a GRID of systems where they can get or set information which is relevant to the situation they are facing or the action they are taking. Such information is not necessarily contained in single systems, but is potentially spread over the network, and is delivered, collected, reconciled and supplied through a distributed, "on demand" integration. WORKPAD's peers will manage internal databases and procedures, will export a schema of their data, will supply query and update methods, will establish and evaluate acquaintances with other systems (mappings), and will support publish\&subscribe mechanisms to allow real-time notification of information updates. The integration logics will be therefore distributed: no specific integration nodes will be required "a priori".

The features of the WORKPAD P2P back-end will represent an enhancement of current centralized federative approaches like those implemented in USA by "The Capital Wireless Integrated Network" (CapWIN, http: //www. capwin.org/). In the scenario of collaborative work of human operators in emergencies, in fact, a peer-to-peer architecture offers a number of advantages:

- autonomy of organizations backing the field teams will be preserved;

- distribution of semantic mappings will ensure a rapid and incremental deployment of the integration logics;

- modularity will ensure that front-end networks will not be bloated with unnecessary data;

- endpoint-based communication will limit impacts of network dynamics (nodes entering or leaving, outages, etc), and will ensure scalability, flexibility, and performances.

In particular, WORKPAD's P2P back-end infrastructure will be designed on a spectrum of industrial standards that includes Web services and GRID Data Access and Integration 


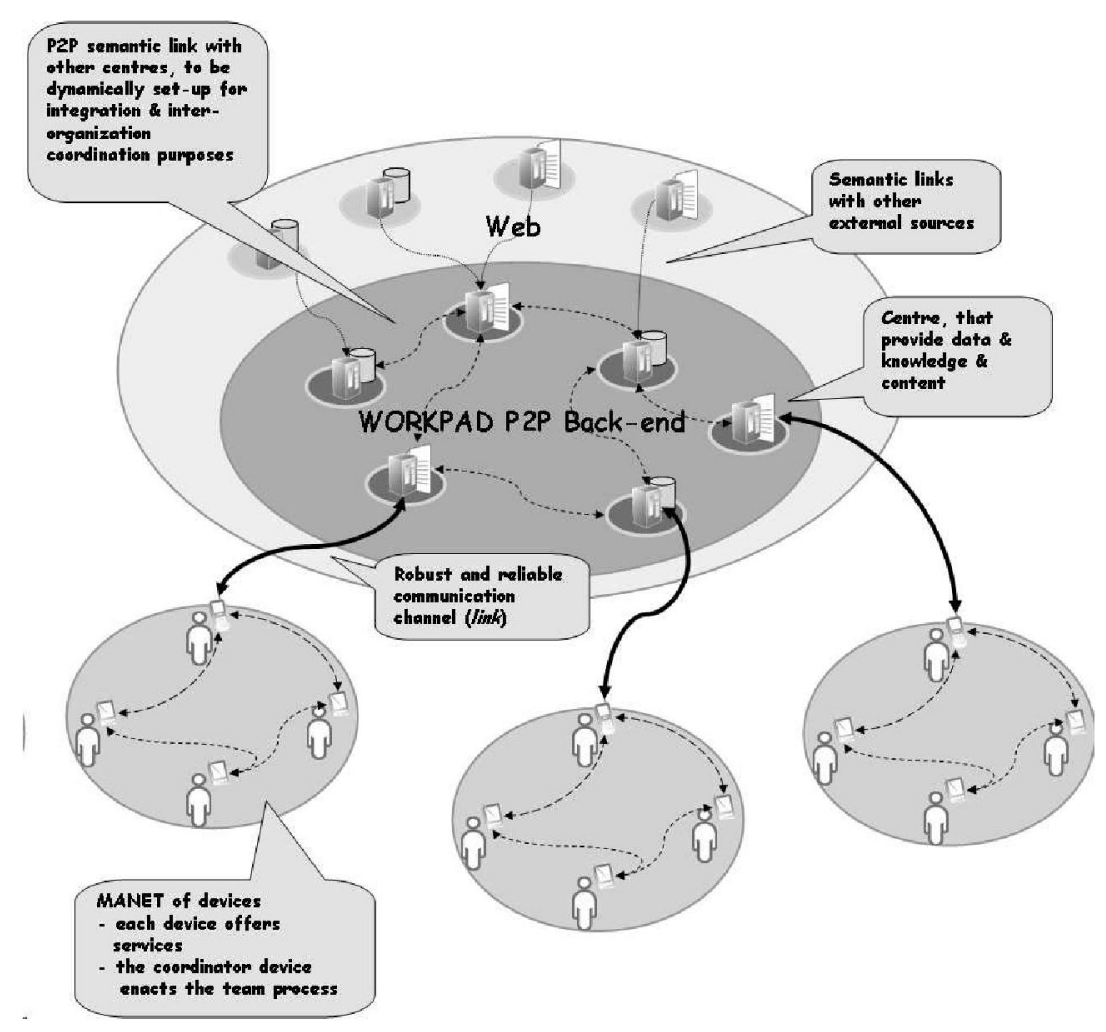

Fig. 3. The WORKPAD 2-layer P2P architecture

services (OGSA-DAI). Specifically, it will be developed as an extension of OGSA-DAI services in the line of the Hyper framework [7]. This will grant support to the widest range of existing platforms, will allow leveraging on all relevant available technologies, and will ensure minimal impacts on legacy systems.

Front-end. The innovative approach that will be adopted can be represented as a model comprised of three levels, and additional definable roles assigned to each level:

- The Human-interaction Level deals with the actual people and parties involved in an emergency scenario (e.g. policemen, fire brigades, paramedics, etc.) which are mostly assigned to rather strict hierarchies and structures within their organisations. They usually have a clear division of roles within the members of a team. Possible roles occurring on this layer would be: team leader, team member, members with special skills, etc. Those roles are mostly assigned once and they do not change during the emergency event except in specific, exceptional conditions.

- The Architecture Level deals with the entities involved in such a mobile environment and with the communication between those, which require a great flexibility. For this, a dynamic mechanism of assigning the different roles has to be implemented which is independent from the roles assigned to the involved people (i.e., on the Human Level). The basic aspect of this level is the capability to adaptively manage disconnection/task anomalies, in order to correctly manage the MANET formed by the team members and the enacted processes.

- The Hardware\&Communication Level includes deployed hardware such as smart phones, satellite-based terminals, PDAs, tablet-PCs, notebooks, etc. Moreover, it shall be possible to cover various transmissions technologies and provide a mechanism to choose the most appropriate one, which is available at a time. The choice will be based on parameters such as bandwidth, transfer rate, costs, reliability, availability, etc.

These three levels are correlated. The roles defined at one level are not necessarily the same at all three levels. For example, a team leader of a fire brigade working on a portable laptop will be the leader of the team on the human level, but will not necessarily be the leader on the architectural level. In the project we will deal with all the three levels of the model, although our specific research focus can be seen mostly in the architecture level.

Figure 4 shows the supposed pervasive architecture for the front-end teams, which comprises four layers: (i) Coordination Layer, (ii) Prediction Layer, (iii) Network Service Interface and (iv) Wireless Stack.

Each device has a wireless stack consisting of a wireless network interface and other technologies for positioning and calculating distances from other devices. On top, a network service interface offers to upper layers the basic services for sending and receiving messages (through multihop paths) to and from other devices, by abstracting over the specific rout- 


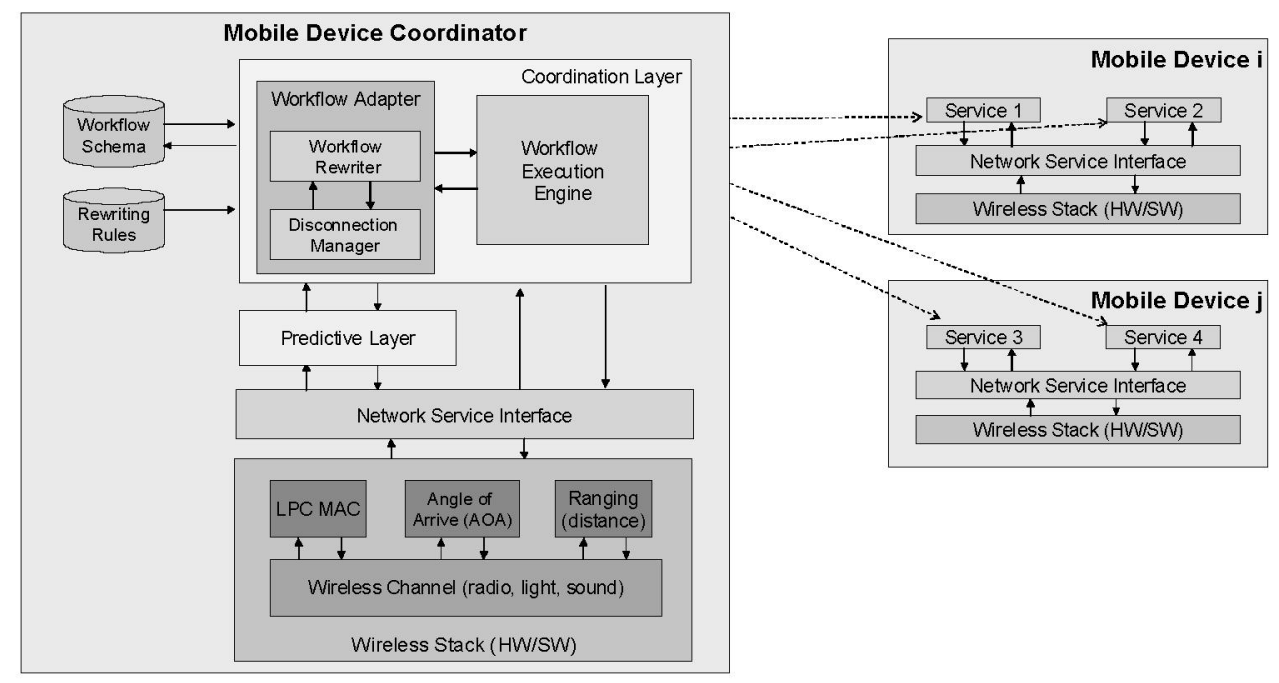

Fig. 4. The proposed pervasive architecture for WORKPAD front-end

ing protocols. Offered services (that is, specific applications supporting tasks of the devices' human users) are accessible to other devices and can be coordinated and composed cooperatively. Some of these services are applications that do not require human intervention (for example, an image-processing utility). Others act as proxies for humans. For example, the service for instructing human users to follow a peer is a GUI that alerts the user by displaying a pop-up window on his or her device and emitting a signal. On top of the network service interface, the coordinator presents the predictive layer, which signals probable disconnections to the coordination layer. The coordination layer manages situations when a peer is going to disconnect (for example, by signalling a specific device to "follow peer $x$ "). For example, if the coordination layer implements a workflow management system, then, on the basis of the current prediction, the coordination layer might restructure the workflow schema. The coordination layer is also in charge of maintaining communications with the backend and accessing integrated data/information \& content.

Preliminary results on the adaptive process management has been presented in [8], in which a restructuring algorithm is proposed and has been implemented in a real software prototype experimented on a simulation platform ${ }^{2}$.

An innovative aspect that will be considered for the adaptiveness of the front-end is the role of geo-information. Indeed geo-referenced data play an increasingly important role in emergency management scenarios, e.g., origins and destinations of messages, assessment of place at risk, location of an accident, allocation of the rescue teams all involve geographic information. Acquisition, use, and integration of geo-information with wide range of seemingly unrelated information are crucial in environmental and emergency situations; they represent a basis for decision making in collaborative

\footnotetext{
${ }^{2}$ The interested reader can take a look at a demonstration video available at: http://www.dis .uniromal.it/ deleoni/ documents/DMCVideo.avi.
}

teams. The result is a growing need for infrastructures that support the access and exchange of geo-information. The issues of geo-information appear on the back-end and front-end side. The back-end processing usually requires sophisticated server and GIS software functionalities, and often contains a substantial amount of spatial data combined with other data, specific for the organisation involved in emergency activities. This complexity hampers the exchange of geo-information. The integration of $\mathrm{P} 2 \mathrm{P}$ technology and the proliferation of location-aware mobile devices may help to lower the barrier for participation and enable new forms of collaboration. Research is needed to understand the basis for collaborative interactions with geo-information, particularly when access rights and expertise vary widely among the members of a team. A higher level of geo-collaboration, beyond simply sharing geographic data, is required to support these kinds of efforts.

Adaptive collaboration patterns. At the Human-interaction Level, in order to define processes to be adaptively enacted, the key idea is to develop and perform interaction mining in current human-to-human collaborations, human-to-service collaborations and service-to-service collaborations, to collect log data. Based on this log data, by applying process/interaction mining algorithms, we can detect and derive new collaboration patterns among humans and software applications [9]. This newly found patterns could then be used to pro-actively aggregate new software services/organizations that can be valuable and reusable for further collaborative tasks. In such a way, the contribution of the project will be not only at the software infrastructure level, but also in the devising of innovative ways of collaborating in emergency situations.

Connecting front-end and back-end. In order to achieve an integrated infrastructure where front-end nodes can have access to a back-end, the MANET should be connected to wired networks with cellular, satellite or TETRA solutions acting as routers. An asymmetrical MANET is a MANET where there are some special nodes (e.g., the coordinator device) 
representing a gateway to the fixed network. Such nodes should be equipped with at least two network interfaces, one connected to the MANET and the other one to the back-end, and should be able to receive and return traffic from outside hosts and route it to the destination MANET node. Such devices play a critical role and become bottlenecks, therefore WORKPAD will research how to design an effective connection depending on the number of nodes, available bandwidth on the uplink interface, priority levels of information, available networks. Moreover, some possibilities for the link front-end/back-end will be studied and experimented, in order to choose the most suitable one (cfr. Figure 5):

- The satellite solution. Satellite communication systems are becoming more readily available, and are used when requiring either the wide bandwidth to support the transmission of video, and other critical data or need to maintain $100 \%$ coverage across a wide area. Satellite services are however not a cost effective solution when the need is just to transmit voice and data within one country. The existing problem is also that the connection of the satellite (the most available in disaster scenarios) and MANET bring together the worst of the two worlds when considering link characteristics: long delays and strongly correlated packet losses from the satellite.

- The UMTS (Universal Mobile Telecommunication System) solution. UMTS represents an evolution in terms of capacity, data speeds and new service capabilities from second generation mobile networks. Today, more than 60 3G/UMTS networks using WCDMA technology are operating commercially in 25 countries. 3G/UMTS has been specified as an integrated solution for mobile voice and data with wide area coverage.

- The TETRA (Terrestrial Trunked Radio) solution. TETRA technology is a world standard published by ETSI for digital mobile trunking networks. TETRA networks offer secure, digital mobile communication for professional organizations such as oil and gas companies, utilities, transportation and public safety and security sector (police, emergency, civil defence, security services, etc.). Data communication improves field operations by allowing users to access databases via their radios. As of today, TETRA provides voice and data services efficiently because it has data capabilities integrated into its infrastructure and is supported by standard TETRA terminals. This is an economical alternative to providing dedicated radio resources for data. TETRA will be investigated in WORKPAD in order to see the feasibility of using it, by comparing coverage and data transfer rates.

\section{RELATED WORK}

In the last years, many projects and initiatives, especially in USA and in Europe, have investigated many issues related to the ones considered by WORKPAD.

In particular, some projects consider mobile devices and their adoption in emergencies. Specifically:
- SHARE $^{3}$ develops advanced mobile services to support rescue forces during their operation (includes audio, video, text \& graphics as well as location information).

- LIAISON ${ }^{4}$ is focused on Location Based Services (LBS) for the enhancement of working environments, based on the Galileo infrastructure. Among the scenarios for validation are fire brigade intervention, and incident management.

- AMIRA $^{5}$ improves access and resources available to support urgent and critical diagnostics and decision making by mobile field workers in safety critical events.

- POMPEI ${ }^{6}$ aims to develop an integrated architecture and a mobile application suite for advanced services, to assist mobile teams of safety/security and emergency workers in crisis management and response. The system comprises workflow management models running on off-theself state-of-the-art mobile phones utilizing sophisticated, appropriately extended operating systems by Symbian.

- ORCHESTRA ${ }^{7}$ aims at developing a Unified Open Architecture for Risk Management Applications.

- MOS AIC ${ }^{8}$ tries to build scenarios and roadmaps for mobile and location-aware working environments.

- RIMSAT $^{9}$ considers integrated innovative knowledge engineering techniques and their validation in a safety-critical environment.

- $\mathrm{X}-\mathrm{MOB}^{10}$ develops a Pan-European ASP-Oriented Open Mobile Platform, validated in scenarios including emergency healthcare application and access to emergency command \& control.

- WEARIT@WORK ${ }^{11}$ proves the applicability of computer systems integrated to clothes, the so-called wearables, in various industrial environments. These novel computer systems will support their users or groups of users in an unobtrusive way wearing them as a computer-belt. This will allow them to perform their primary task without distracting their attention enabling computer applications in novel fields. Interaction with wearables by the user must be minimal to realize optimal system behaviour. For this reason a wearable computer recognizes by integrated sensors the current work progress of a user. Based on the work context detected the system pushes useful information to its user, e.g., how to proceed with the work. Apart from speech output, media could be optical systems presenting the information, e.g., via semitransparent glasses within the workers visual field. Output devices for tactile feedback will be applicable, too. Notably, the Paris Fire Service and Rosenbauer Intl, maker of fire service and

\footnotetext{
${ }^{3}$ http: //www.ist-share.org/

${ }^{4}$ http://liaison. newapplication.it/index.html

${ }^{5}$ http: //www amira.no/

${ }^{6}$ http://www .pompei-eu.com/

${ }^{7}$ http: //www.eu-orchestra.org/

${ }^{8}$ http://www mosaic-network.org/

${ }^{9}$ http://www.rimsat.com/

${ }^{10}$ http://www.xmob-project.org/

${ }^{11}$ http: //www. wearitatwork.com/
} 


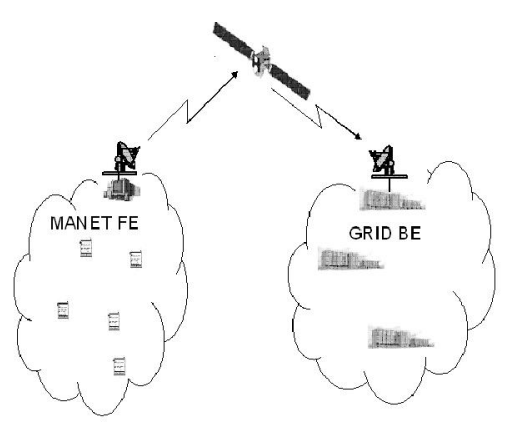

(a) Satellite-based link

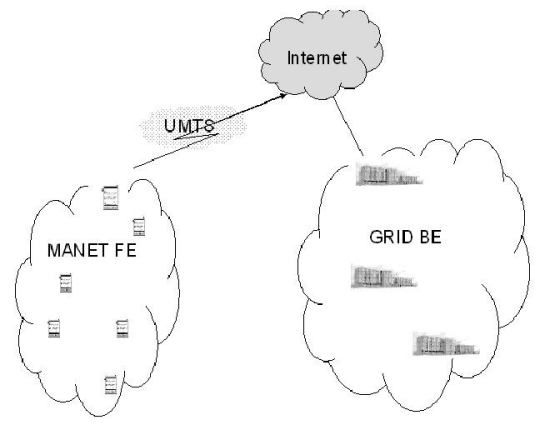

(b) UMTS-based link

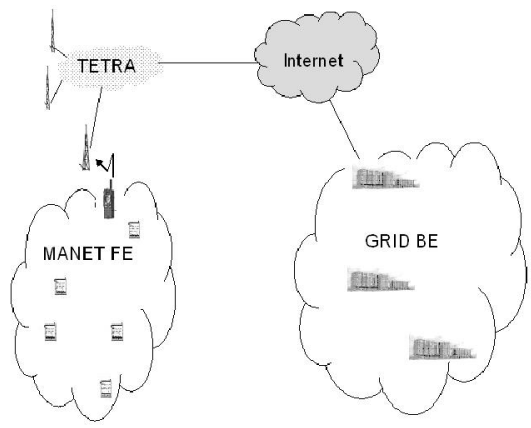

(c) TETRA-based link

Fig. 5. Possible alternatives for the FE/BE link

rescue equipment, are among the partners.

- FORMIDABLE ${ }^{12}$ aims to propose a European Standard Methodology for Natural Hazards Management based on the consensus of major Mediterranean Civil Protections, and to develop an interoperable support system prototype which integrates the resulting guidelines.

- EGERIS ${ }^{13}$ provides Civil Protection organisations (and different actors in Emergency Management) with Information and Communication technologies that improve their overall efficiency during the preparedness and the response phases of a crisis.

But none of them adopts a peer-to-peer paradigm, being based on more traditional architectures.

A few projects have considered the issue of data integration in peer-to-peer settings. Among them, (i) PIAZZA ${ }^{14}$ aims to provide "semantic mediation" between an environment of thousands of peers, each with its own schemas, rather than requiring the use of a single, uniform, centralized mediated schema to share data between peers, and (ii) TERRAWORLD 15 is one of the first research initiatives in the field of geointegration. Moreover, (iii) RAMFLOOD ${ }^{16}$ is an example of a decision-making system using combined, mixed data resources

\footnotetext{
${ }^{12}$ http://www . formidable-project.org/

13 http://www .egeris .org/

${ }^{14}$ http://data.cs.washington.edu/p2p/piazza/

15http://www.isi.edu/integration/Terraworld/

${ }^{16}$ http://www. cimne.com/ramflood/
}

gathered from earth observation, satellite positioning systems, in-situ sensors and geo-referenced information with advanced graphical visualisation methods for gathering knowledge.

\section{CONCluding Remarks}

This paper has introduced WORKPAD, a system to provide a 2-layered workspace suitable in emergency scenarios. The WORKPAD approach to New Working Environments (NWEs) is based on the interplay between emergency networks and collaborative nomadic teams on one side and geo/work-data and content integration on the other side.

The project started on September 1st, 2006, and has a duration of 36 months, comprising 8 partners and 9 workpackages. It will release the first scientific innovative results within month 18 and a prototype of the system, to be then validated by the users, withing month 30 .

The most innovative aspects of the WORKPAD system are: $\mathrm{P} 2 \mathrm{P}$ data and content integration, adaptive workflow management services and geo-collaboration. The project will produce middleware and software components to improve the development of specific emergency IT infrastructures. At the same time, new paradigms of peer-to-peer communication and information integration will be developed and refined in a relevant and challenging applicative scenario. Hence, the strategic impact of this project is twofold: on one hand, the application of new technologies will help addressing the new and increasing threats to public security; on the 
other hand, the development of an innovative solution for emergency operations will improve the state of the art in several IT emerging disciplines enabling the interoperability among different teams belonging to different organisations (fire brigades, national security, national guard, civil protection units, etc.). For that reason, the benefit we expect from this project consists in both the improvement of Homeland Security capabilities and the progress of IT industry. The availability of a specific IT and data infrastructure for emergencies can significantly improve the quality of the intervention of firstresponders. Moreover, it can effectively assist in coordinating rescue teams and operations in the first crucial days after the emergency/disaster event. Finally, it can help planning and performing recovery activities in later emergency/disaster management phases. In particular, thanks to its innovative P2P architecture, the WORKPAD framework will give the ability of integrating a mobile wireless network to a rich back-end information network in a flexible, cost-effective, reliable, and powerful manner.

Acknowledgements. This work is supported by the European Commission through the FP6-2005-IST-5-034749 project WORKPAD.

\section{REFERENCES}

[1] A. Zerger, D.I. Smith. Impediments to using GIS for Real-time Disaster Decision Support. Computers, Environment and Urban Systems, vol. 27 (2), pp. 123-141, 2003.

[2] S.H. Leitinger. Comparision of GIS-based Public Safety Systems For Emergency Management. Proc. 24th Urban Data Management Symposium, 2004.

[3] GIS for Emergency Management. http://www.esri.com/library/ whitepapers/pdfs/emermgmt.pdf, prompted on Sept. 20, 2005.

[4] E. Plate, B. Merz (eds.). Naturkatastrophen: Ursachen, Wirkung, Vorsorge. Schweizerbart'sche Verlagsbuchhandlung, Stuttgart, 2001.

[5] F. De Rosa, A. Malizia, and M. Mecella. Disconnection Prediction in Mobile Ad hoc Networks for Supporting Cooperative Work. IEEE Pervasive Computing, vol. 4 (3), 2005.

[6] E. Galanti. Metodo Augustus - Guida del Dipartimento della Protezione Civile. http://ww. casaleinforma.it/ pcivile/scarica/04augustus .pdf, 2004 (in Italian).

[7] D. Calvanese, G. De Giacomo, M. Lenzerini, R. Rosati, G. Vetere. Hyper: A Framework for Peer-to-Peer Data Integration on GRIDs. Proc. Semantics for GRID Databases, Ist International IFIP Conference on Semantics of a Networked World (ICSNW 2004), LNCS 3226, 2004.

[8] M. de Leoni, F. De Rosa, M. Mecella. MOBIDIS: A Pervasive Architecture for Emergency Management. Proc. 4th International Workshop on Distributed and Mobile Collaboration (DMC 2006).

[9] S. Dustdar, R. Gombotz. Discovering Web Service Workflows using Web Services Interaction Mining. Forthcoming on International Journal of Business Process Integration and Management, 2006. 